\title{
Multiple Velocity Profile Measurements in Hypersonic Flows Using Sequentially-Imaged Fluorescence Tagging
}

\author{
Brett F. Bathel* \\ University of Virginia, Hampton, Virginia, 23681 \\ Paul M. Danehy ${ }^{\dagger}$, Jennifer A. Inman ${ }^{\ddagger}$, Stephen B. Jones ${ }^{\S}$ \\ NASA Langley Research Center, Hampton, Virginia, 23681-21991 \\ Christopher B. Ivey ${ }^{* *}$ \\ Johns Hopkins University, Baltimore, MD, 21218 \\ and \\ Christopher P. Goyne $\mathrm{i}^{\dagger \dagger}$ \\ University of Virginia, Charlottesville, Virginia, 22904
}

\begin{abstract}
Nitric-oxide planar laser-induced fluorescence (NO PLIF) was used to perform velocity measurements in hypersonic flows by generating multiple tagged lines which fluoresce as they convect downstream. For each laser pulse, a single interline, progressive scan intensified CCD camera was used to obtain separate images of the initial undelayed and delayed NO molecules that had been tagged by the laser. The CCD configuration allowed for sub-microsecond acquisition of both images, resulting in sub-microsecond temporal resolution as well as sub-mm spatial resolution $(0.5-\mathrm{mm} \times 0.7-\mathrm{mm})$. Determination of axial velocity was made by application of a cross-correlation analysis of the horizontal shift of individual tagged lines. Quantification of systematic errors, the contribution of gating/exposure duration errors, and influence of collision rate on fluorescence to temporal uncertainty were made. Quantification of the spatial uncertainty depended upon the analysis technique and signal-to-noise of the acquired profiles. This investigation focused on two hypersonic flow experiments: (1) a reaction control system (RCS) jet on an Orion Crew Exploration Vehicle (CEV) wind tunnel model and (2) a 10-degree half-angle wedge containing a 2-mm tall, 4-mm wide cylindrical boundary layer trip. The experiments were performed at the NASA Langley Research Center's 31-inch Mach 10 wind tunnel.
\end{abstract}

\section{Introduction}

$\mathrm{T}$ HE ability to perform quantitative velocity measurements in a hypersonic flow can be complicated by the extreme dynamic and thermodynamic conditions present in hypersonic test facilities and the limited optical access to the test section. To date, off-body measurements performed in the NASA Langley Research Center's 31inch Mach 10 facility have been limited to Schlieren imaging, ${ }^{1}$ pressure rake measurements, ${ }^{2}$ and more recently nitric oxide planar laser-induced fluorescence (NO PLIF) flow visualization. ${ }^{1}$ The velocity measurements described in this paper were all performed in this facility using the molecular tagging velocimetry (MTV) technique. ${ }^{3,4}$ The MTV technique is a variation of the PLIF technique, which has already been demonstrated to be an effective off-

\footnotetext{
* Graduate Student, National Institute of Aerospace/University of Virginia, MS 493, AIAA Student Member.

${ }^{\dagger}$ Research Scientist, Advanced Sensing and Optical Measurement Branch, MS 493, AIAA Associate Fellow.

${ }^{\ddagger}$ Research Scientist, Advanced Sensing and Optical Measurement Branch, MS 493, AIAA Member.

$\S$ Technician, Advanced Sensing and Optical Measurement Branch, MS 493.

** Undergraduate Student, Mechanical Engineering, 223 Latrobe Hall 3400 N. Charles St., AIAA Student Member.

${ }^{\dagger}$ Research Assistant Professor, Mechanical and Aerospace Engineering, Aerospace Research Laboratory, PO Box 400248, AIAA Associate Fellow.
} 
body flow visualization tool capable of providing spatially-resolved qualitative flowfield information. The NO PLIF technique is well suited for use in Langley's 31-inch Mach 10 facility due to the optical access and the availability of externally accessed pressure taps through which a gaseous seeding system can be attached to the facility.

By slightly modifying the existing NO PLIF optical setup within this facility, we have been able to perform two separate MTV experiments providing spatially resolved, quantitative velocity information. The first experiment provided velocity data for a yaw reaction control system (RCS) jet on an Orion Crew Exploration Vehicle (CEV), described in Reference 6. In this experiment, a spatial comb filter placed in the path of the laser sheet created a series shadows to provide velocity profiles. The second experiment provided boundary layer velocity data for a 10degree half-angle wedge model, described in References 7 and 8 . The velocity profiles for this experiment were generated using a diffusion welded cylindrical lens array consisting of 251 -m focal length cylindrical lenses sandwiched together. For both experiments, the nominal freestream Mach number was approximately 9.68 with a unit Reynolds number of approximately $1.7 \times 10^{6} /$ meter $\left(0.5 \times 10^{6} /\right.$ foot $)$.

The purpose of this paper is to both report the demonstration of the MTV technique as applied to two differing hypersonic flows in a large-scale wind tunnel facility and to describe the analysis technique used to determine the velocity profiles and the associated measurement uncertainties. Prior work using the MTV technique has been performed by a number of authors using various techniques to analyze the fluorescence profiles. One particular analysis method, involving the cross-correlation of image pairs, was first described by Gendrich and Koochesfahani ${ }^{9}$ to calculate 2-dimensional velocity profiles with sub-pixel accuracy. This paper loosely follows the method outlined by these researchers; however it has been adapted for a one-component velocity measurement. The work reported in this paper makes use of an intensified, double-shutter camera with sequential frame spacing of $500 \mathrm{~ns}$, which removes measurement sensitivity to vibration. The camera used in this work also eliminates errors that are otherwise associated with a two-camera velocimetry measurement (scaling and shifting the two separately acquired images). This paper also provides for the first time an estimation method to correct the velocity profiles obtained to compensate for fluorescence decay and its effect on the spatial shift obtained via the cross-correlation method. To the authors' knowledge, this paper represents the first presentation of single-shot velocity measurements with quantified uncertainty using the NO MTV technique.

\section{Experimental Setup}

The complete experimental setup consisted of the optical setup, camera system, two wind tunnel models, the PLIF diagnostic system, and the wind tunnel facility. Discussion of the two wind tunnel models, the PLIF diagnostic system and the wind tunnel facility will be summarized briefly. The camera system and its timing methodology will be discussed in detail in Section II.D.

\section{A. Wind Tunnel Facility}

The 31-Inch Mach 10 Air Tunnel is an electrically-heated blowdown facility located at NASA Langley Research Center in Hampton, Virginia, USA. The full details of this facility can be found in the paper by Micol, ${ }^{10}$ a brief summary of which is provided here. The facility has a nominal Mach number of 10 and a 31-inch square test section and operates on electrically heated, compressed air. Large windows, transparent in the ultraviolet, form three walls (including top, side and bottom) of the test section with the fourth wall formed by the model injection system. The top window allows for the laser sheet to pass through the tunnel section, while the side window allows for imaging of the flow region of interest. The model is side-mounted to the fourth wall. Run durations for the current tests were about one minute. A single facility stagnation pressure, $\mathrm{P}_{0}$, of $2.41 \mathrm{MPa}(350 \mathrm{psia})$ was investigated for both configurations. The nominal stagnation temperature was 1,000 K (1,800 Rankine) for the experiments described herein. Based upon the stagnation conditions, the freestream Mach number was 9.68, the freestream velocity was $1404 \mathrm{~m} / \mathrm{s}$, the freestream pressure was $68.3 \mathrm{~Pa}\left(9.90 \times 10^{-3} \mathrm{psi}\right)$, and the freestream temperature was $52.3 \mathrm{~K}(94.2$ Rankine). ${ }^{10}$

\section{B. Wind Tunnel Models}

The first experiment was conducted using an Orion Crew Exploration Vehicle (CEV) model. ${ }^{6}$ The axis of symmetry of the model was oriented at an $18^{\circ}$ angle of attack with respect to the freestream velocity. In this test, only a single yaw RCS jet was analyzed. The RCS jet fluid consisted of a mixture of approximately $5 \%$ NO and $95 \% \mathrm{~N}_{2}$ and was supplied to the pre-nozzle chamber at two pressures, $0.31 \mathrm{MPa}$ (45 psi) and $0.91 \mathrm{MPa}$ (132 psi), so as to simulate two different jet operating conditions. 
The second experiment was conducted using a $10^{\circ}$ half-angle wedge model with a sharp leading edge, similar to that described in Reference 7. A 2-mm tall, 4-mm wide cylindrical boundary layer trip was positioned along the centerline 75.4-mm downstream of the leading edge. The NO was $100 \%$ pure, supplied at 150 SCCM, and was seeded into the laminar boundary layer from an 11-mm-long, 0.81-mm-wide slot located 29.4-mm downstream of the leading edge along the centerline. The flowrate supplied through this slot was assumed not to have perturbed the boundary layer flow. This assumption is based upon surface heat transfer measurements performed by Berry et al. ${ }^{11}$ using a similar gas supply configuration. Analysis of these heat transfer measurement results and the boundary layer perturbation associated with the present wedge model are discussed by Danehy et al. ${ }^{8}$ A 2-mm-tall, 4-mm-diameter cylindrical protuberance located 75.4-mm downstream of the leading edge along the centerline deflected the laminar boundary layer gas.

\section{PLIF System}

A pulsed Spectra Physics Pro-230 Nd:YAG laser was used to pump a Sirah Cobra Stretch dye laser at 532-nm to achieve a 622-nm output beam. This output was mixed with 355-nm light from the Nd:YAG in a Sirah Frequency Conversion Unit to produce $\sim 5 \mathrm{~mJ}$ per pulse at $226-\mathrm{nm}$. This beam was directed to the sheet-forming optics affixed above the tunnel test section. The duration of the pulse at this wavelength was approximately $10 \mathrm{~ns}$.

To form a laser sheet, the collimated 226-nm beam was passed through a 36-mm focal length cylindrical lens, which diverged the beam, expanding the beam in one direction while leaving it collimated in the other. A 1-m spherical lens then collimated the diverging axis of the beam and focused the other axis into a thin sheet approximately $75-\mathrm{mm}$ wide by $0.5-\mathrm{mm}$ thick. To tag multiple lines of NO in the test section for a velocimetry measurement, a spatial comb filter was placed below the sheet-forming optics. For the Orion CEV test, this filter was used to create the series of vertical shadows. For the wedge model, a 50-mm-long, LaserOptik diffusion welded lens array of 25, 1-m focal length cylindrical lenses focused the laser sheet into 25 lines, running parallel to the model surface in the spanwise direction. The lens array had an anti-reflection coating. The lines formed with each method typically had widths of approximately $0.85-\mathrm{mm}$ at FWHM, with a 2-mm separation distance between each line.

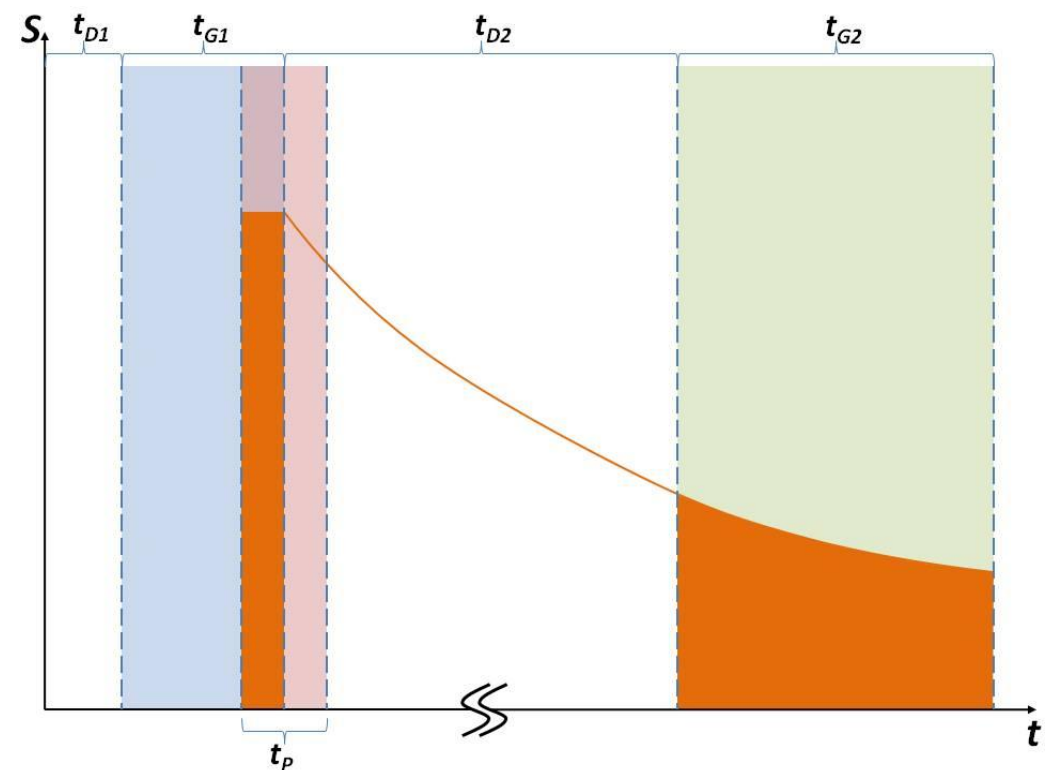

Figure 1. Camera and laser timing sequence and signal intensity (orange regions).

\section{Camera System and Timing}

To image the tagged lines in both experiments, a Cooke Dicam Pro camera, utilizing an intensified 1280x1024 pixel array interline progressive scan $\mathrm{CCD}$, was used. For the wedge model, only a $1280 \times 256$ pixel region was used to image the flow so that an image read-out rate of $10 \mathrm{~Hz}$, corresponding to the laser pulse frequency, could be achieved. Similarly, a 1280x288 pixel region was used for the CEV experiment. A 105-mm focal length, F/4.5 Nikon lens was used for the CEV experiment and a 100-mm focal length, F/2.0 B. Halle lens was used for the wedge experiment. Spatial resolutions achieved with this camera were 17.3 pixels $/ \mathrm{mm}$ (439.4 pixels/inch) and 17.5 pixels $/ \mathrm{mm}$ ( 444.5 pixels/inch) for the CEV and wedge model experiments, respectively. When used in double shutter mode, the camera is capable of acquiring an image pair with a minimum $500 \mathrm{~ns}$ delay between the end of the first gate and the beginning of the second. Each gate has a minimum duration of $20 \mathrm{~ns}$, with delay settings and durations set in increments of $20 \mathrm{~ns}$.

After tagging the $\mathrm{NO}$ gas, the fluorescence intensity decays exponentially in time. The rate of this decay depends upon the local pressure, temperature, and gas composition of the tagged molecules. The camera used in these 
experiments did not allow for independent gain settings for the first and second exposures. To ensure a measurable signal during the second exposure, it was necessary to use the minimum gate delay setting of $500 \mathrm{~ns}$ and keep the second gate open long enough to collect an adequate number of photons. Additionally, to maintain comparable signal intensities in both the first and second CCD exposures the signal in the first exposure had to be attenuated. This was achieved by using the shortest gate possible ( $20 \mathrm{~ns})$. Additionally, the timing delay of the first gate was such that it collected only the first several nanoseconds of the laser pulse. This overlap of the first exposure and laser pulse is achieved by simultaneously triggering the camera and laser, both of which obtained trigger signals from a Lab Smith LC-880. This overlap of the first camera gate and the laser pulse is referred to as the effective first gate, having a duration of $t_{e G I}$. By adding varying lengths of coaxial cable between the camera trigger input and the LC880 , adjustments on the order of $1 \mathrm{~ns}$ are made on the delay of the first gate.

Table 1. Laser and camera timing parameters.

\begin{tabular}{|c|c|}
\hline \multicolumn{2}{|c|}{ Laser } \\
\hline Pulse Duration, $t_{P}$ & $10 \mathrm{~ns}$ \\
\hline & Camera \\
\hline $1^{\text {st }}$ Delay, $t_{D 1}$ & Arbitrary \\
\hline $1^{\text {st }}$ Gate, $t_{G 1}$ & $20 \mathrm{~ns}$ \\
\hline Effective $1^{\text {st }}$ Gate, $t_{e G 1}$ & $5-10 \mathrm{~ns}$ \\
\hline $2^{\text {nd }}$ Delay, $t_{D 2}$ & $500 \mathrm{~ns}$ \\
\hline $2^{\text {nd }}$ Gate, $t_{G 2}$ & $100 \mathrm{~ns}($ Wedge) \\
& $300 \mathrm{~ns}(\mathrm{CEV})$ \\
\hline \multirow{2}{*}{ Resolution } & $\begin{array}{c}1280 \times 256 \text { pixels @ } 17.5 \text { pixels } / \mathrm{mm} \text { (Wedge) } \\
1280 \times 288 \text { pixels @ } 17.5 \text { pixels } / \mathrm{mm} \text { (CEV) }\end{array}$ \\
\hline
\end{tabular}

Table 1 gives the timing parameters used in the experiments. Figure 1 shows a generalized timing sequence adopted for these experiments. The vertical axis represents signal intensity, with the orange line showing the exponential decay behavior of fluorescence in time. The orange regions represent the timevarying signal intensity measured during the first and second exposures. The blue and green regions are indicative of the first and second gate durations, respectively, while the red region indicates the laser pulse duration.

\section{Velocity Analysis}

Determination of the axial velocity is based upon a time-of-flight calculation in which the measured distance between the center of mass of the profile observed in the undelayed and delayed images, $\Delta x$, is divided by the time separating their acquisition, $\Delta t$. The velocity relation is therefore:

$$
V=\frac{\Delta x}{\Delta t}
$$

Obtaining values for $\Delta x$ and $\Delta t$ will be described in sections B and $\mathrm{C}$, respectively.

\section{A. Image Pre-Processing}

The spatial resolution for both experiments was obtained by capturing an image consisting of a matrix of square marks separated at equal spatial intervals, known as a 'dotcard' and described in Reference 12, which was in the plane of the laser sheet used to interrogate the seeded NO gas.

To correct for optical and perspective distortion of the images in the wedge experiment, the image of the dotcard in the test section was acquired with the camera and a corresponding undistorted image of the same dotcard was created with Adobe Acrobat software. An image registration algorithm, UnwarpJ ${ }^{13}$ was then used to correct the distortion. The image registration algorithm was not applied to the RCS velocity experiment images.

To improve the signal-to-noise ratio in the images, a 4-pixel radius average disk filter was applied to the undelayed and delayed fluorescence images. The images were then binned by 4 pixels in the vertical direction. The image registration, mean filtering, and pixel binning were performed with ImageJ, a freeware image processing program available from National Institutes of Health. ${ }^{14}$ This provided an approximate spatial resolution of $0.5-\mathrm{mm}$ by $0.7-\mathrm{mm}$.

Prior to processing each image for velocity information, a background offset level was obtained from images taken with the laser blocked and subtracted from the undelayed and delayed images. Due to the shortness of the gate delay used between the undelayed and delayed images (500 ns) and the associated decay time of the P46 phosphor in the intensifier, some ghosting remained from the undelayed image in the delayed image. For the $500 \mathrm{~ns}$ gate delay, this level was experimentally determined to be approximately $1 / 126^{\text {th }}$ of the undelayed image. Therefore, this additional fraction of the undelayed image was subtracted from the delayed image in addition to the normal background offset level. 


\section{B. Spatial Analysis}

To obtain the relative shift between individual lines in the undelayed and delayed image, a window was selected that encompassed each vertical tagged line in the undelayed image. For each row of pixels in this window, a undelayed fluorescence profile was obtained. An additional window was positioned encompassing the same pixel region in the delayed image, with the delayed fluorescence profiles again acquired for each row in the window. A user defined threshold, based upon the maximum pixel intensity for each row in the undelayed image, was used to reject low signal profiles which might otherwise provide poor quality velocity information. In this case, no velocity information is reported.

For each remaining profile pair, each of length $L$, a cross-correlation was performed using MATLAB $®$ 's 'xcorr' function. The maximum value resulting from the array (of length $2 L+1$ ) of cross-correlation coefficient values was then found and a $7^{\text {th }}$-order polynomial fit, similar to the method used by Gendrich and Koochesfahani, ${ }^{9}$ centered about this maximum, was obtained. For this analysis, 11 points (centered about the maximum) were used to compute the polynomial fit. Setting the first derivative of this polynomial equal to zero and using a root-finding algorithm to determine the maxima of the polynomial fit, the resolved maximum of the coefficient values was obtained. The resulting difference between this resolved maximum and the length of the input profile vector, $L$, corresponds to the total shift, in pixels, of each profile pair.

To obtain the average spatial displacement, in time, at a particular location along a tagged profile in the flowfield, the mean spatial shift for a set of $N$ image pairs was calculated.

\section{Timing Simulation and Analysis}

In the absence of fluorescence decay, a phenomenological geometric argument can be made, based upon the camera and laser timing parameters selected, to describe the elapsed time between the undelayed and delayed images. This approach is a refinement of that of Danehy et al. ${ }^{4}$ Figure 2 shows a space-time-signal diagram describing an infinitesimal spatial width laser beam exciting fluorescence at a rate of 1 block of fluorescence per

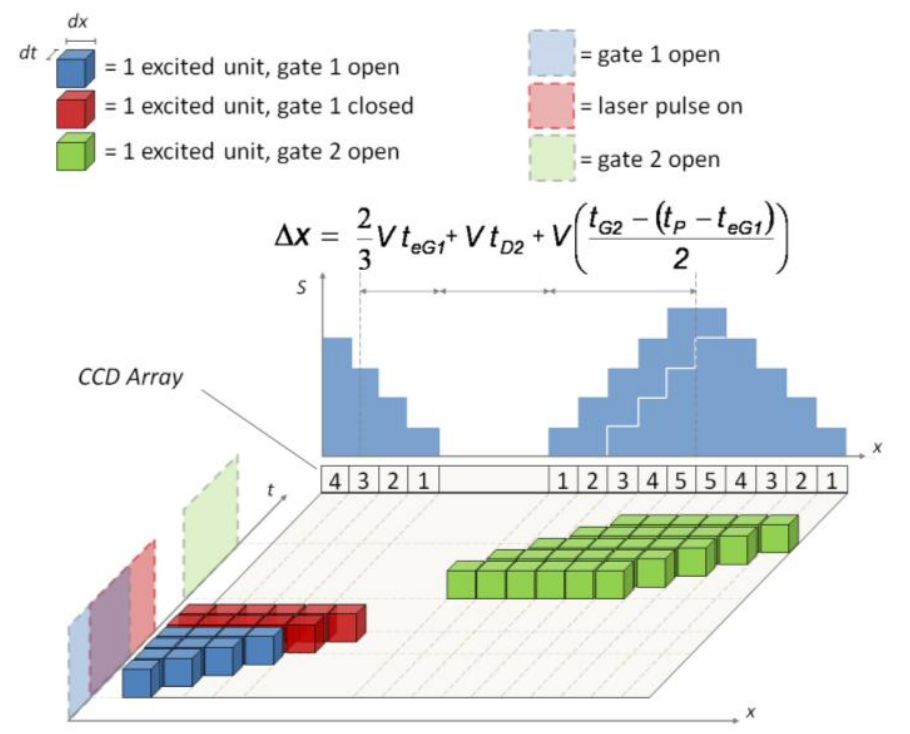

Figure 2. Space-time-signal diagram of idealized CCD signal and acquisition of fluorescence. The time axis (left) shows the gate and laser timing windows. In this figure, the first gate and laser pulse windows overlap. The colored blocks represent excited nitric oxide volumes in time and space. The rear axis gives the spatial signal profile across the CCD. unit of time. The axes are $x$ for space, $t$ for time, and $S$ for PLIF signal intensity.

The blocks, which represent a 1dimensional region of NO gas, are color coded according to the means by which they emit fluorescence photons. The blue blocks are excited and emit photons captured by the CCD camera during the overlap of the first camera gate and the laser pulse. The red blocks are excited and emit fluorescence while the laser pulse continues, but after the first camera gate has been closed (that is, they are not captured by the first camera gate). The light green blocks represent fluorescence captured during the second camera gate. The left-hand axis in Figure 2 shows the occurrence of the first gate, laser pulse, and second gate, which are represented by the shaded areas with dashed borders.

During the laser pulse, if a non-zero axial velocity is present, each block of NO gas is assumed to be excited instantaneously by the pulse and convected one spatial unit, $\Delta x$, downstream per unit of time, $\Delta t$, with the ratio corresponding to the axial velocity, $V=\Delta x / \Delta t$. 
At each time step in this process, the emitted fluorescence from each block is captured by a pixel on the camera CCD array. During the first camera gate, the blocks of NO gas are excited by the laser and then convected downstream. The accumulated charge distribution developed on the pixels corresponding to captured photons emitted by these blocks takes on a right triangle-like profile. In Figure 2, this charge distribution is represented by the left-hand, light-blue shaded area on the signal-spatial axis. The numbers, located directly below this area, are segmented into pixels along an array of the CCD and represent the total charge accumulated (in arbitrary units) during the first exposure. After the first camera gate has closed, a total of four blocks of $\mathrm{NO}$ gas have been excited by the laser pulse. As the rectangular-shaped laser pulse continues for an additional two time units, and additional two blocks of NO gas are excited, resulting in a train of excited NO gas that is a total of six blocks long.

At the end of the laser pulse, the six blocks of NO gas are convected downstream over a period of time corresponding to the difference of second gate delay duration and the duration of the laser pulse firing after the end of the first camera gate. After this period of time, the second camera gate is opened and the six block train of NO gas convects downstream while the fluorescence is again captured by the CCD pixels. In this case, the charge distribution across the pixels takes on a trapezoidal shape, as shown in the right-hand, light-blue shaded area on the signal-spatial axis in Figure 2. Note that if the end of the laser pulse had coincided with the end of the first camera gate, the NO gas would have been a 4-block train, then the profile shape observed in the second camera gate would instead appear to be narrower and weighted to the right. This alternate profile shape is represented by the light-blue shaded area falling under the solid white segmented line.

Based upon this timing sequence, the perceived distance between the center of mass of the fluorescence profiles acquired during the first and second camera gates is:

$$
\Delta x=V \cdot\left(\frac{2}{3} t_{e G 1}+t_{D 2}+\frac{t_{G 2}}{2}-\frac{\left(t_{P}-t_{e G 1}\right)}{2}\right)
$$

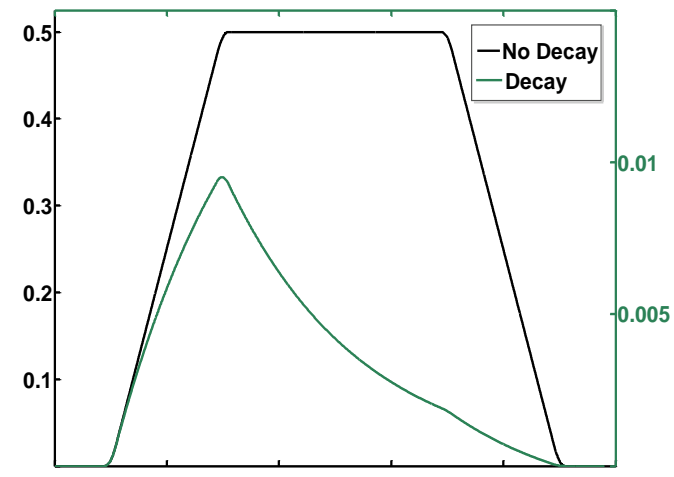

(a)

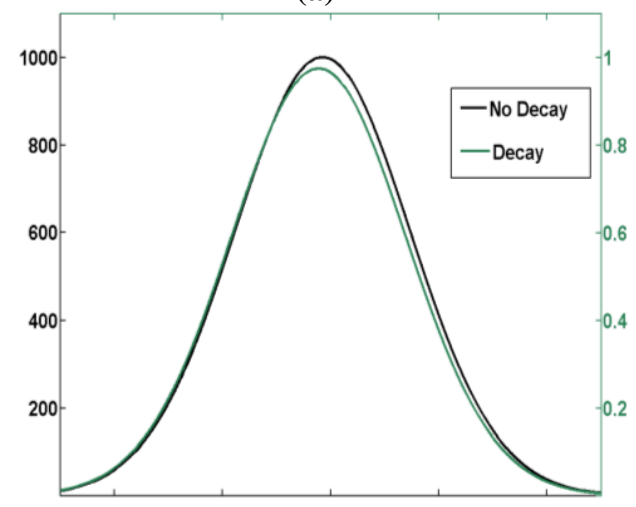

(b)

Figure 3. Simulated CCD exposure using (a) a narrow top-hat spatial laser profile and (b) Gaussian spatial laser profile. Scales are in arbitrary units.
In this example, $2 t_{e G 1} / 3$ corresponds to the center of mass of the right triangle profile observed in the first exposure, $t_{D 2}$ is the delay between the end of the first and start of the second exposures, $t_{G 2} / 2$ is the center of mass of the profile observed in the second exposure, and $-\left(t_{P}-t_{e G 1}\right) / 2$ corresponds to the added weighting of the second exposure profile due to the laser pulse occurring after the first gate. By dividing both sides of the equation by the velocity, $V$, the net elapsed time between the first and second camera gates is obtained.

When considering the fluorescence decay during the camera gate, the perceived center of mass of the fluorescence profile observed in the delayed image becomes slightly left-weighted. This is because the fluorescence intensity of the tagged gas region decays exponentially in time. Therefore, as the gas is convected downstream, the distribution of photons collected across the CCD pixel array diminishes with the decay of the tagged NO molecules, with the upstream side of the pixels receiving more fluorescence than the downstream pixels. Figure 3a shows a simulated CCD exposure in which a spatially narrow top-hat beam is used to excite NO molecules being convected from left to right. This simulated second exposure, occurring $500 \mathrm{~ns}$ after the end of the first gate, shows the resulting fluorescence profiles observed with (green) and without (black) the effect of decay. The simulation is exaggerated to emphasize that an error can result if care is not taken to compensate for the effect of fluorescence decay. Figure 3b shows a more realistic simulation involving a 16-pixel wide (FWHM) Gaussian beam profile with (green) and without (black) fluorescence decay. 
If the fluorescence lifetime is known, this perceived shift in the center of mass of the fluorescence profile can be estimated by simulating the fluorescence acquired by both camera exposures. By performing such a simulation, a relation between the maximum signal intensities of the undelayed and delayed frames and the fluorescence lifetime can be formulated.

However, if the fluorescence lifetime is not known, a simulation using the experimental camera timing parameters can be used to estimate the profile shape for different lifetimes. Based upon the signal ratios, the lifetime can be inferred and used to compensate for the perceived profile shift.

This approach was used in this paper to estimate the profile shifts observed experimentally. A range of fluorescence lifetimes corresponding to conditions encountered during typical wind tunnel tests were simulated for three different second-gate durations: $100 \mathrm{~ns}, 200 \mathrm{~ns}$, and $300 \mathrm{~ns}$. The simulation follows the process described in Figure 2, but using finer spatial and temporal step sizes. The data points show representative values explicitly calculated with $80 \mathrm{~ns}, 100 \mathrm{~ns}, 150 \mathrm{~ns}$, and $192.6 \mathrm{~ns}$ lifetimes.

For these simulations, the gas velocity was fixed at $1000 \mathrm{~m} / \mathrm{s}$ and $t_{P,} t_{e G I}$, and $t_{D 2}$ were fixed at $10 \mathrm{~ns}, 5 \mathrm{~ns}$, and 500 $\mathrm{ns}$, respectively. A fine temporal spacing of $\Delta t=0.05 \mathrm{~ns}$ was used with a corresponding grid spacing of $\Delta x=0.02$ $\mu \mathrm{m}$. The spatial grid spacing corresponded to a pixel discretization of $3.5 \times 10^{-4}$ experimentally. During the first simulated exposure, the exciting beam position was fixed at a particular spatial location. For each time step, the intensity distribution was added to a profile array (initially an array of zero values), which was then shifted by $\Delta x$, and the process repeated for the duration of the laser pulse. This profile array was simultaneously added to the first exposure array (also initially an array of zero values) during each time step until the end of the effective first gate, $t_{e G I}$. The profile array was then shifted to account for the delay between the first and second gate as shown in Figure 2. For the second exposure, the profile array was added to the second exposure array during each time step until the end of the second gate, $t_{G 2}$. At each time step in the full simulation, the profile array was multiplied by the exponential decay factor:

$$
\begin{gathered}
\exp \left[-\frac{m \cdot(\Delta t)}{\tau_{L I F}}\right] \\
\tau_{L I F}=\frac{1}{A_{21}+Q(P, T)}
\end{gathered}
$$

where $m$ is the current time step, $Q$ is the pressure/temperature-dependent quenching rate and $A_{2 l}$ is the spontaneous emission rate, both given by Settersten et al. ${ }^{15,16}$ as $1 / 192.6 \mathrm{~ns}^{-1}$.

After the first and second simulated exposures with and without fluorescence decay were obtained, the highly resolved exposures were artificially pixilated to reflect the resolution of the experiment. The cross-correlation algorithm described in the previous section was then applied to the simulated first and second artificially pixilated exposures, both with and without fluorescence decay, to determine the relative shift. The ratio of relative spatial shift, $\Delta x$, to the fixed velocity, $V=1000 \mathrm{~m} / \mathrm{s}$, provided the elapsed time between gates. By comparing the cases with and without fluorescence decay, a correction factor was obtained characterizing the relation between both cases:

$$
\Delta t=C \cdot \Delta t_{\mathrm{No} \mathrm{Decay}}=C \cdot\left(\frac{2}{3} t_{e G 1}+t_{D 2}+\frac{t_{G 2}}{2}-\frac{\left(t_{P}-t_{e G 1}\right)}{2}\right)
$$

where the correction factor, $C$, was determined by analyzing the ratio of maximum signal intensity between the undelayed, $S_{1, \max }$, and delayed, $S_{2, \max }$, images. The ratio of intensity values, $S_{1, \max } / S_{2, \max }$, which is a measure of the fluorescence lifetime of NO, can be used to infer the magnitude of shift due to decay. Using the results of the simulation, the following relation between signal intensity ratio and correction factor was obtained using TableCurve 2D®:

$$
C=a+b\left(\frac{s_{1, \max }}{s_{2, \text { max }}}\right)+c\left(\frac{s_{1, \max }}{s_{2, \text { max }}}\right)^{3 / 2}+d \log \left(\frac{s_{1, \max }}{s_{2, \text { max }}}\right)+e \frac{100}{\left(\frac{s_{1, \max }}{s_{2, \text { max }}}\right)^{2}}
$$

where the coefficient values for $a, b, c, d$, and $e$ vary with the second gate duration. Figure 4 shows the behavior of this correction factor as a function of fluorescence lifetime. Curves for second gate durations of $100 \mathrm{~ns}, 200 \mathrm{~ns}$, and $300 \mathrm{~ns}$ are shown. 


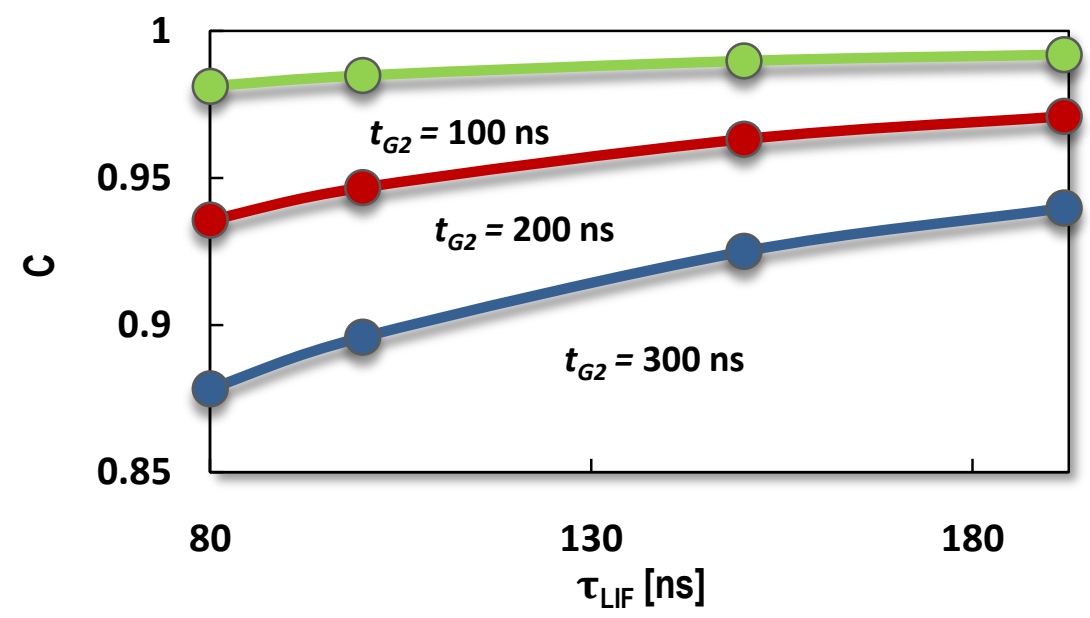

Figure 4. Variation of timing correction factor as a function of fluorescence lifetime. Results are based upon simulation using 16-pixel-wide Gaussian spatial laser profile.

\section{Uncertainty Analysis}

The velocity uncertainty was based upon the contributions of magnification, accuracy errors, errors associated with spanwise velocity components, and the contributions of spatial and temporal uncertainties. The general form of the velocity uncertainty is:

$$
u_{V}=u_{V, \text { Magnification }}+u_{V, \text { Accuracy }}+u_{V, \text { Spanwise }}+\sqrt{u_{V, \Delta x}^{2}+u_{V, \Delta t}^{2}}
$$

\section{A. Spatial Uncertainty}

The uncertainty in velocity due to spatial uncertainties, $u_{V, \Delta x}$, was obtained by first determining the spatial shift, $\Delta x_{i}$, along each fluorescence profile for each single-shot image. The single-shot and average spatial uncertainties at $95 \%$ confidence for a particular location were determined, respectively, by analyzing the sensitivity of the measured velocity to variations in spatial shift:

$$
u_{V, \Delta x}=\frac{\partial V}{\partial(\Delta x)} \cdot u_{\Delta x}=\frac{1}{\Delta t} \cdot t_{N-1,95 \%} \sqrt{\frac{\sum_{i=1}^{N}\left(\Delta x_{i}-\overline{\Delta x}\right)^{2}}{N-1}}=\frac{t_{N-1,95 \%} \cdot \sigma_{\Delta x}}{\Delta t}, u_{V, \overline{\Delta x}}=\frac{u_{V, \Delta x}}{\sqrt{\bar{N}}}
$$

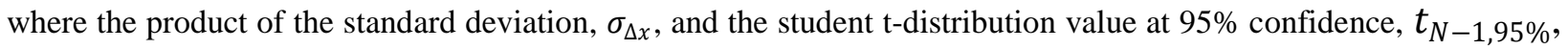
represents the uncertainty in each spatial and temporal variable.

\section{B. Timing Uncertainty}

The uncertainty in velocity due to uncertainties in the time separation, $\Delta t$, includes systematic uncertainties resulting from camera and laser timing jitters and duration uncertainties as well as uncertainty due to variations in signal intensities, which are used to infer the lifetime and calculate the correction factor. The definition of the uncertainty in velocity due the timing separation is:

$$
\boldsymbol{u}_{V, \Delta t}=\frac{\partial V}{\partial(\Delta t)} \cdot \boldsymbol{u}_{\Delta t}
$$

and the net temporal uncertainty, $\boldsymbol{u}_{\Delta t}$, can be defined as:

$$
u_{\Delta t}=\sqrt{u_{\Delta t_{C}}^{2}+u_{\Delta t_{D 1}}^{2}+\left(\frac{2 \cdot C \cdot u_{\Delta t_{e G 1}}}{3}\right)^{2}+\left(C \cdot u_{\Delta t_{D 2}}\right)^{2}+\left(\frac{C \cdot u_{\Delta t_{G 2}}}{2}\right)^{2}+\left(\frac{C \cdot u_{\Delta t_{P}}}{2}\right)^{2}+\left(\frac{C \cdot u_{\Delta t_{e G 1}}}{2}\right)^{2}}
$$


where the uncertainty due to correction factor, $\boldsymbol{u}_{\Delta t_{C}}$, is defined below. The remaining timing uncertainties are defined in Table 2.

On a shot-to-shot basis, variation in the signal intensity between the undelayed image and delayed image at a point in the tagged profiles resulted in a variation in the correction factor. This resulted in apparent variations to the elapsed time between images. To minimize the magnitude of this variation, the second gate duration could be decreased. This was done in the wedge model experiment. In Figure 4, as the second gate duration is decreased, the magnitude of correction factor decreases along with its slope as a function of fluorescence lifetime, which can be inferred experimentally from the signal intensity ratio. The single-shot uncertainty associated with the correction factor, $\boldsymbol{u}_{\Delta t_{C}}$, at a point is calculated in the following equations.

$$
u_{\Delta t_{C}}=\frac{\partial(\Delta t)}{\partial C} \cdot u_{C}=\left(\Delta t_{N o \text { Decay }}\right) \cdot u_{C}
$$

where $u_{C}$ is the uncertainty in the correction factor. Further expanding this term gives:

$$
\begin{aligned}
& \boldsymbol{u}_{C}=\frac{\partial C}{\partial\left(s_{1, \max } / S_{2, \max }\right)} \cdot \boldsymbol{u}_{\left(s_{1, \max } / S_{2, \max }\right)} \\
& \boldsymbol{u}_{\left(s_{1, \max } / s_{2, \max }\right)}=\sqrt{\left(\frac{\partial\left(s_{1, \max } / s_{2, \max }\right)}{\partial s_{1, \max }} \cdot u_{s_{1, \max }}\right)^{2}+\left(\frac{\partial\left(s_{1, \max } / S_{2, \max }\right)}{\partial s_{2, \max }} \cdot u_{S_{2, \max }}\right)^{2}} \\
& u_{S_{l, \max }}=t_{N-1,95 \%} \sqrt{\frac{\sum_{i=1}^{N}\left(S_{l, \max }-\overline{S_{l, \max }}\right)^{2}}{N-1}} \quad l=1,2
\end{aligned}
$$

where $u_{C}$ is based on the functional form of the correction factor, $C$, in Equation 7.

The average correction factor uncertainty for a set of $N$ images was determined in a similar manner:

$$
\begin{gathered}
u_{\overline{\mathrm{C}}}=\frac{\partial C}{\partial\left(S_{1, \max } / S_{2, \max }\right)} \cdot u_{\overline{S_{l, \text { max }}}} \\
u_{\overline{S_{l, \text { max }}}}=\frac{u_{S_{l, \max }}}{\sqrt{N}} \quad l=1,2
\end{gathered}
$$

Table 2. Camera and laser timing uncertainties.

\begin{tabular}{|c|c|}
\hline \multicolumn{2}{|c|}{ Timing Uncertainties } \\
\hline Laser Pulse Jitter, $u_{\Delta t_{P}}$ & $\pm 0.1 \mathrm{~ns}$ \\
\hline $1^{\text {st }}$ Delay Jitter, $u_{\Delta t_{D 1}}$ & $\pm 2.0 \mathrm{~ns}$ \\
\hline Effective $1^{\text {st }}$ Gate Duration, $u_{\Delta t_{e G 1}}$ & $\pm 1.0 \mathrm{~ns}$ \\
\hline $2^{\text {nd }}$ Delay Jitter, $u_{\Delta t_{D 2}}$ & $\pm 0.5 \mathrm{~ns}$ \\
\hline $2^{\text {nd }}$ Gate Duration, $u_{\Delta t_{G 2}}$ & $\pm 1.0 \mathrm{~ns}$ \\
\hline
\end{tabular}

The variation in the camera and laser timing also contributed to the temporal uncertainty. Table 2 gives the magnitude of camera and laser timing and jitters and duration uncertainties used in the uncertainty analysis, with the largest contribution being from the jitter in the delay of the first gate. This jitter affected the overlap of the first gate and the laser pulse, as shown in Figure 1, by varying the start of the first gate. Incorporation of these uncertainties is given in Equation 11. The timing and spatial uncertainties are grouped in the rootsum-squared term in Equation 8, and represent the uncertainty in velocity precision.

\section{Magnification Uncertainty and Velocity Accuracy}

To determine the uncertainty due to the magnification, several resolution measurements were taken at ten different locations on the dotcard images. The average magnification and the standard deviation of the magnification, $\sigma_{\text {Magnification }}$, for the CEV and wedge model tests is given in Table 3 . The general form of the magnification uncertainty for both experiments, using 10 measurements of resolution ( 9 degrees-of-freedom) at $95 \%$ confidence was:

$$
u_{\mathrm{V}, \text { Magnification }}=\frac{\partial V}{\partial R} u_{\text {Magnification }}=\frac{(\Delta x)_{P I X E L}}{\Delta t} \cdot \sigma_{\text {Magnification }} \cdot t_{9,95 \%}
$$


Table 3. Magnification and accuracy values.

\begin{tabular}{|c|c|}
\hline \multicolumn{2}{|c|}{ Magnification Parameters - CEV } \\
\hline Mean Magnification & $5.75 \times 10^{-2} \mathrm{~mm} /$ pixel \\
\hline Standard Deviation, $\sigma_{\text {Magnification }}$ & $\pm 5.06 \times 10^{-5} \mathrm{~mm} /$ pixel \\
\hline Maximum Uncertainty, $u_{V, \text { Magnification }}$ & $\pm 1.12 \mathrm{~m} / \mathrm{s}$ \\
\hline Magnification Parameters - Wedge \\
\hline Mean Magnification & $5.70 \times 10^{-2} \mathrm{~mm} /$ pixel \\
\hline Standard Deviation, $\sigma_{\text {Magnification }}$ & $\pm 3.67 \times 10^{-5} \mathrm{~mm} / \mathrm{pixel}$ \\
\hline Maximum Uncertainty, $u_{V, \text { Magnification }}$ & $\pm 1.49 \mathrm{~m} / \mathrm{s}$ \\
\hline Velocity Accuracy \\
\hline \multicolumn{2}{|c|}{ Accuracy, $u_{V, A c c}$} \\
\hline
\end{tabular}

where $(\Delta x)_{P I X E L}$ is the pixel shift between profiles in the undelayed and delayed images. This uncertainty scaled linearly with the measured pixel shift between the undelayed and delayed images. Table 3 provides an upperbound estimation of the uncertainty in velocity due to magnification error considering a maximum velocity 790 $\mathrm{m} / \mathrm{s}$ for the CEV experiment and 1260 $\mathrm{m} / \mathrm{s}$ for the wedge experiment.

To estimate the accuracy of the cross-correlation technique as applied

to the CEV and wedge experiments, zero-velocity images were obtained and analyzed with the cross-correlation software. A single tagged line was chosen to calculate velocity from these images. The accuracy from this analysis was determined to be $\pm 22 \mathrm{~m} / \mathrm{s}$. This is shown in Table 3 .

\section{Spanwise Velocity Uncertainty}

In addition to estimating the axial velocity uncertainty, uncertainty due to spanwise velocity components (parallel to the laser beam) must also be included. This uncertainty is a result of gas being convected in the spanwise direction between the undelayed and delayed frames. If a portion of the tagged profile at a particular location has moved in the spanwise direction after the first gate has closed, it will be incorrectly correlated with the portion of the undelayed image at the same spanwise location in the delayed image. This uncertainty will be most notable in regions of the flow with steep axial velocity gradients.

A first estimate of this uncertainty can be obtained by vertically shifting the delayed image relative to the undelayed image and recalculating the axial velocities. The differences between the unshifted velocity values and the shifted velocity values provide an estimate of how much a spanwise velocity component would affect the measured axial velocity uncertainty. By doing this, a first estimate of the maximum axial velocity uncertainty due to a spanwise velocity component for a shift of 8 pixels is a maximum of $58 \mathrm{~m} / \mathrm{s}$ for the wedge model.

To obtain a refined estimate of this uncertainty for the wedge model, it was assumed that the only significant velocity components existed in the axial and spanwise directions. For this analysis, we assumed that any measured deviation from the mean unperturbed axial velocity component was assumed to be due to a spanwise velocity component. To estimate the maximum contribution of a spanwise velocity component to the axial velocity uncertainty, the square of the decreased axial velocity measured in the flow was subtracted from the square of the mean axial velocity measured in the unperturbed region. The square root of this difference corresponds to the maximum spanwise velocity component possible. Multiplying this value by $\Delta t_{\text {No Decay }}$, the pixel shift corresponding to the spanwise component was obtained.

By moving the delayed image up or down by 4 and 8 pixels and correlating it with the original undelayed image, the axial velocity values, in the presence of a uniform spanwise velocity component corresponding to these pixel shifts, is calculated. The difference between the shifted axial velocity values and the unshifted axial velocity values is calculated and represent the uncertainty due to a spanwise velocity component. The difference between the unshifted axial velocity and the mean unperturbed axial velocity is then calculated based upon observed velocity deficits and an estimate of the spanwise pixel shift is made. Using this estimated shift, the uncertainty due to this potential spanwise velocity component is interpolated from 4- and 8-pixel shifted axial velocity uncertainty values. 


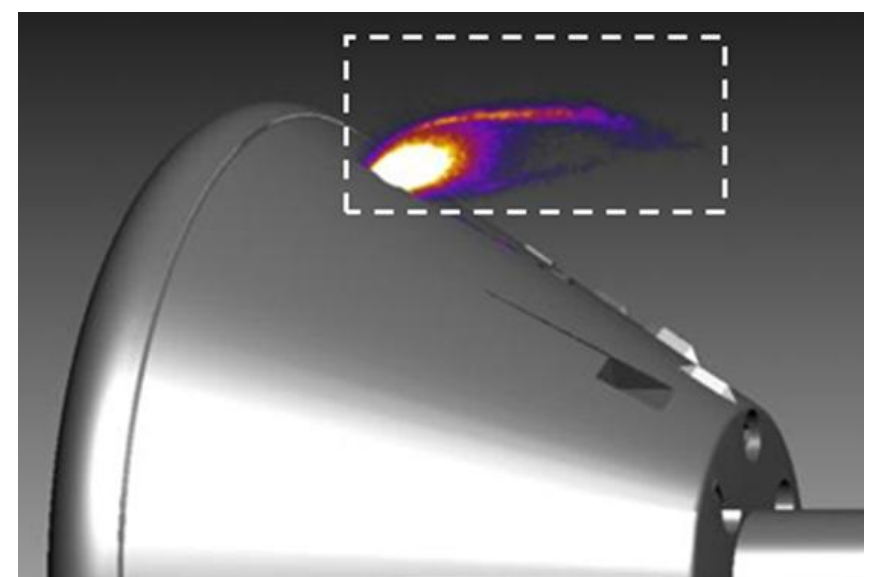

Figure 5. Orion CEV yaw RCS jet and field of view. $A$ false color table has been applied to the PLIF image and is displayed using ViDI.

\section{Results}

\section{A. RCS Jet}

Figure 5 shows a flow visualization image and the corresponding field of view imaged by the camera. Figure $6 \mathrm{a}$ and $6 \mathrm{~b}$ shows an 87-shot average undelayed image and delayed image, respectively. Figure $6 \mathrm{c}$ shows the resulting 87 -shot average velocity components and associated uncertainties superimposed over a single-shot flow visualization image. In Figure $6 \mathrm{c}$, the green ordinate axes are located along the averaged center of mass of the tagged lines in the undelayed image (5a) while the green horizontal axes serve as the velocity scale. The images were created using the Virtual Diagnostics Interface (ViDI) software developed at NASA Langley Research Center. ${ }^{17}$ The software is based upon Autodesk 3DS Max ${ }^{\circledR}$ software and provides a virtual environment in

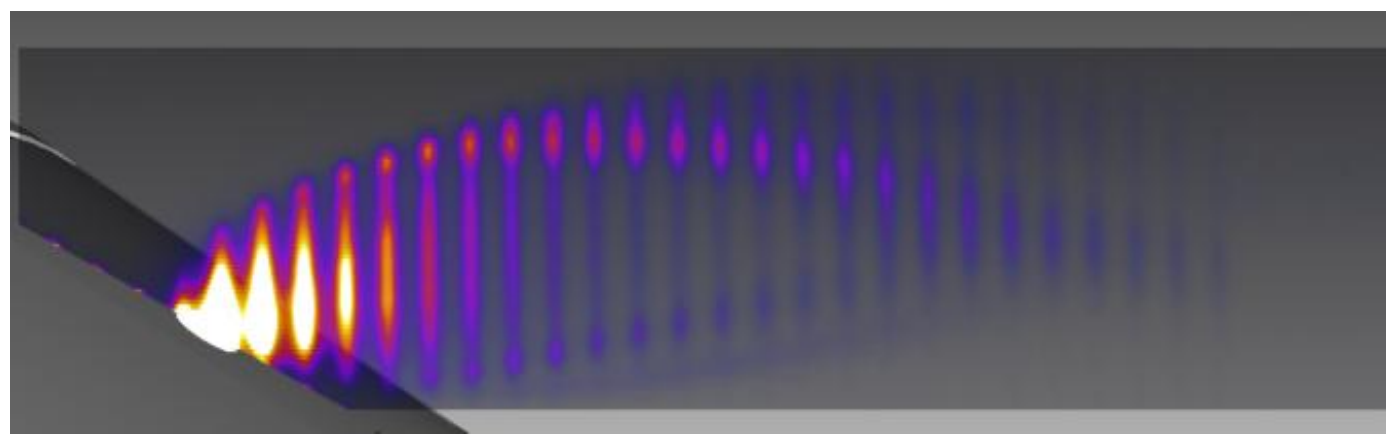

(a)

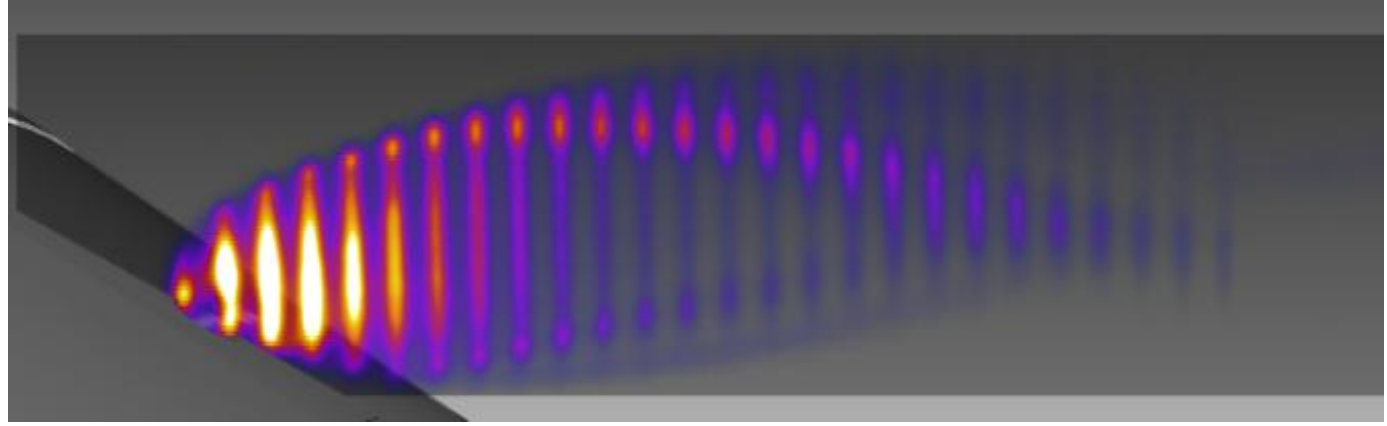

(b)

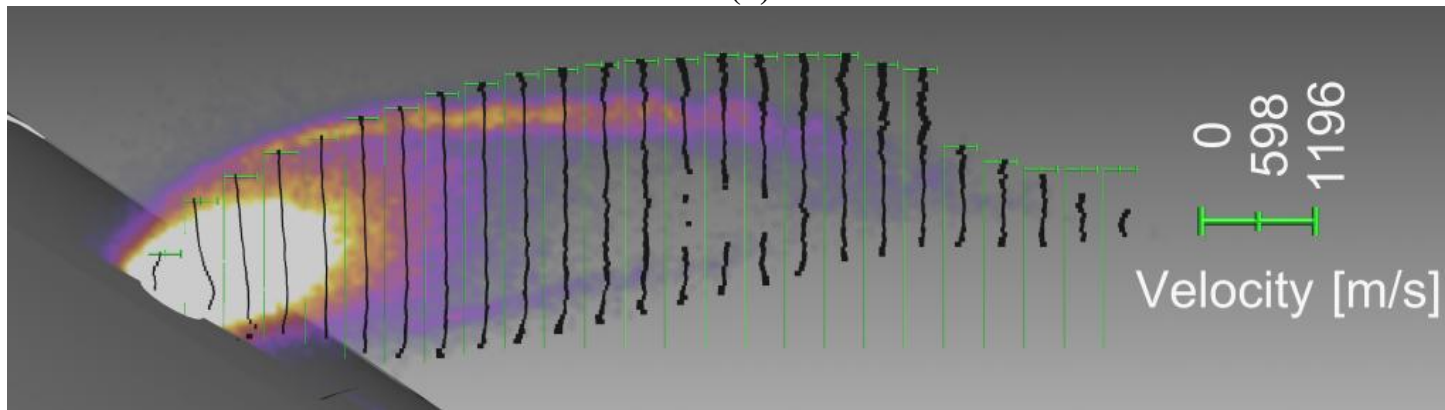

(c)

Figure 6. Orion CEV yaw RCS jet (a) reference tagged image, (b) shifted tagged image, and (c) velocity and uncertainty results superimposed on a flow visualization image. A false color table has been applied to the PLIF and MTV images. 
which experimental data can be combined with virtual models to aid in understanding the related fluid mechanics.

In Figure $6 \mathrm{c}$, the velocity values are represented by the offset of the black points from left-hand vertical axis. The width of these points about the center corresponds to 2-times the total uncertainty (Equation 8). Within the core of the RCS jet, velocities are on the order of $600 \mathrm{~m} / \mathrm{s}$. In the regions of highest signal level, such as the along the midregion of fourth profile from the nozzle, the uncertainties are on the order of 30-40 m/s. In regions where the signal level is relatively low, such as the downstream region of the core, the total uncertainty noticeably increases. This increased uncertainty is due to the difficulty in correlating lower signal-to-noise regions and obtaining a highlypeaked polynomial fit of the correlation coefficients.

Near the downstream and boundary regions of the RCS jet, the jet becomes unsteady and begins to fluctuate in the vertical direction of the image plane. This unsteadiness results in intermittent signal in these regions. The signalto-noise ratio in these regions is also decreased noticeably in comparison to the levels observed at the nozzle exit. These factors result in increased uncertainty. Along the furthest profile from the nozzle, total uncertainties are on the order of $85-115 \mathrm{~m} / \mathrm{s}$.

The most notable deviation from expected velocity occurs along the second profile from the jet exit. The measured velocity of this profile nearest the axis of the nozzle appears to reach a maximum of approximately 1200 $\mathrm{m} / \mathrm{s}$. However, assuming the stagnation enthalpy of the room temperature $\mathrm{N}_{2}$ gas supplied to the RCS jet is completely converted to kinetic energy, the maximum velocity theoretically possible within this jet approximately $790 \mathrm{~m} / \mathrm{s}$. The discrepancy between the measured and theoretical values is attributed to the large fluorescence quenching gradient present near the nozzle exit. For the under-expanded jet, the gas pressure at the exit plane is sufficiently high for quenching to affect the tagged profile. Observing Figure $6 c$, the region nearest the jet exit along the second profile is weighted to the right. Due to this weighting, the cross-correlation performed erroneously calculated a higher velocity at this point. Beyond approximately 2 nozzle exit diameters downstream, this effect is negligible.

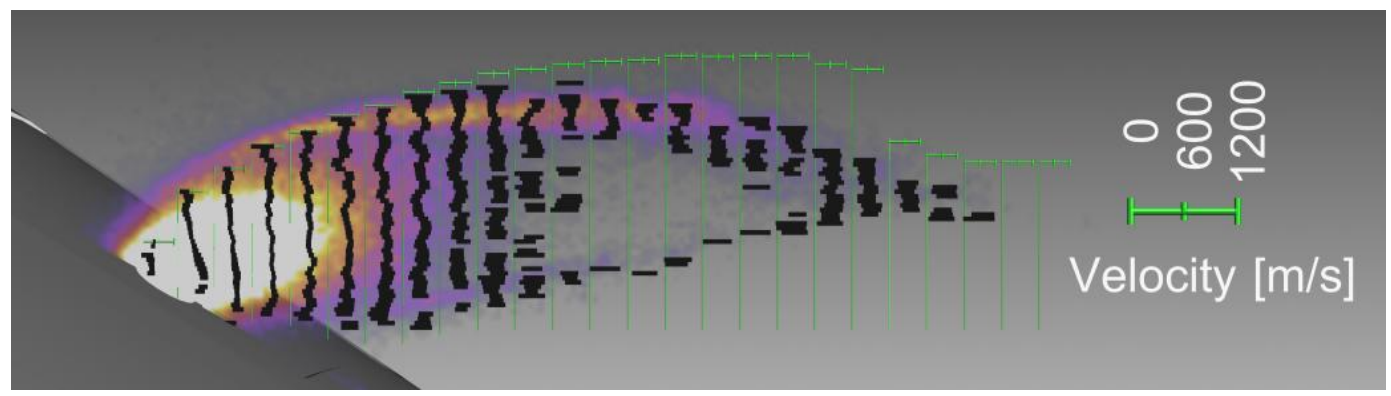

Figure 7. Single-shot yaw RCS jet velocity and uncertainty measurement.

Figure 7 shows a single-shot velocity and uncertainty measurement of the yaw RCS jet. The increased noise level, compared to the noise level for the averaged velocity measurements, occurring in the single-shot images results in greater velocity uncertainties. Similar to the averaged velocity measurement, regions in the single-shot image pair with relatively higher signal-to-noise levels have lower velocity uncertainties than the lower signal-to-

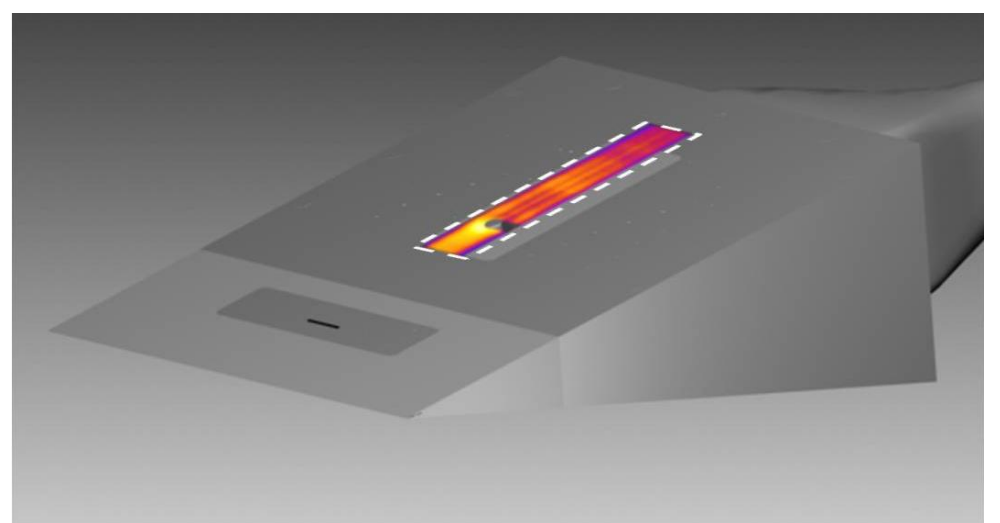

Figure 8. Perspective view of wedge model, trip, and corresponding field of view. noise regions. For the higher signalto-noise regions, such as along the fourth profile from the nozzle exit, the uncertainties are approximately $90 \mathrm{~m} / \mathrm{s}$. For lower signal-to-noise regions, such as along the tenth profile from the nozzle, the uncertainties vary from $170-450 \mathrm{~m} / \mathrm{s}$.

\section{B. Wedge Model}

Figure 8 shows the wedge model with the $2-\mathrm{mm}$ by $4-\mathrm{mm}$ tall cylindrical trip and the corresponding field of view imaged by the camera. Figure 9a shows an averaged PLIF flow visualization image. Figure $9 \mathrm{~b}$ 


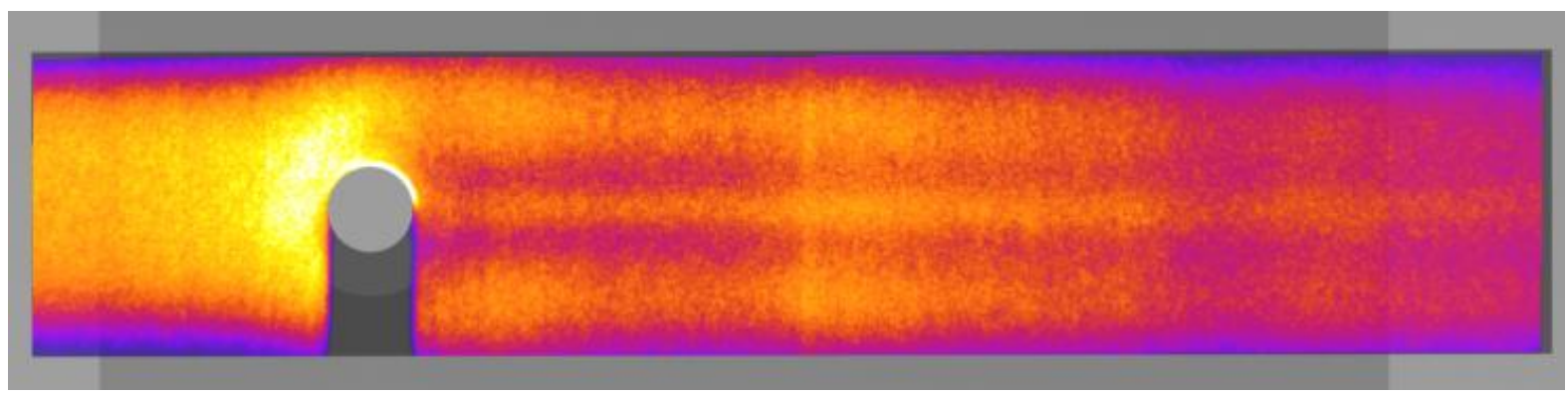

(a)

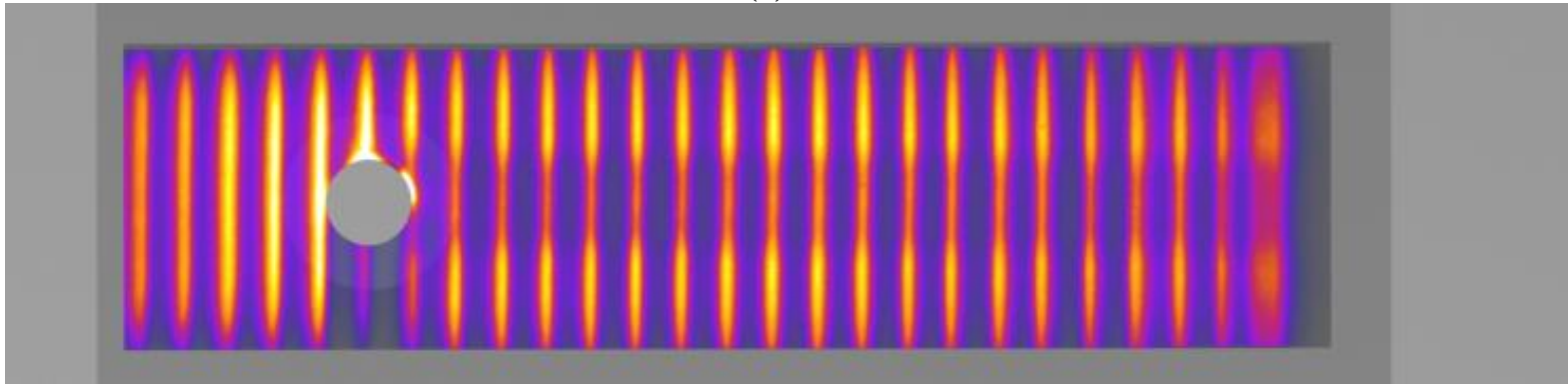

(b)

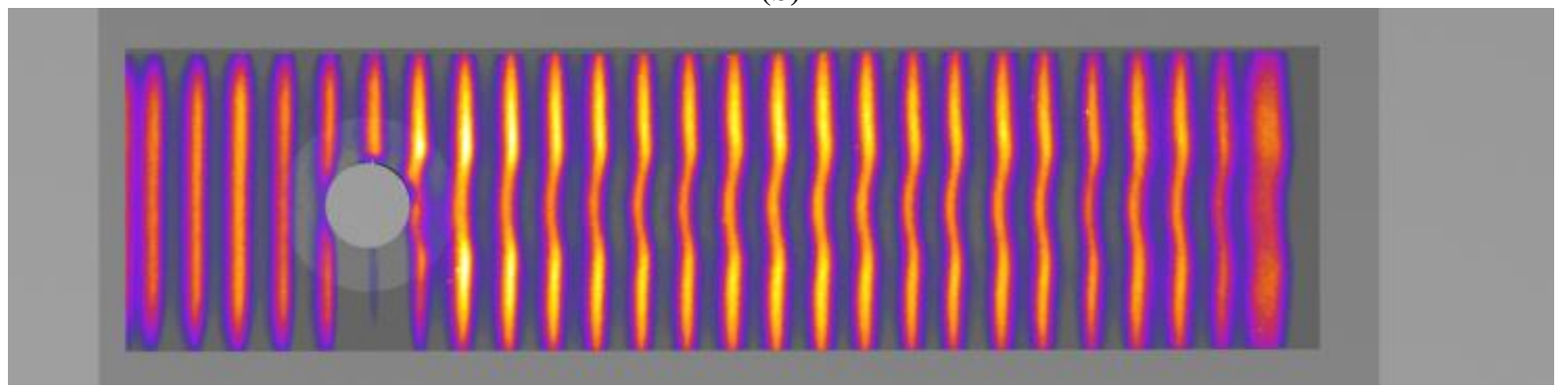

(c)

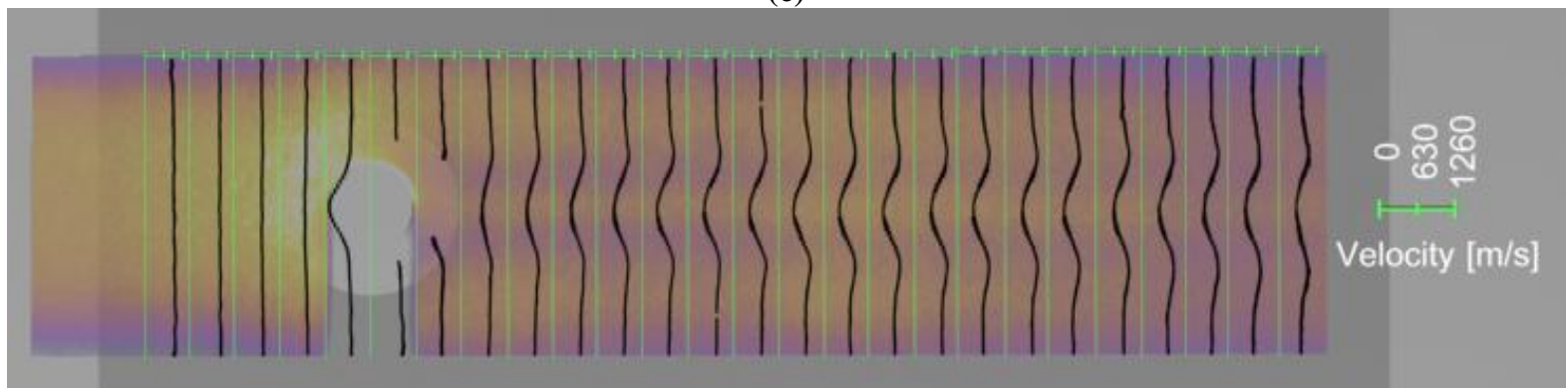

(d)

Figure 9. Wedge model (a) flow visualization image, (b) reference tagged image, (c) shifted tagged image, and (d) velocity and uncertainty results. Velocity scale in (d) is from 0 to $1260 \mathrm{~m} / \mathrm{s}$. The solid lines in (e) are the $41-$ shot mean velocity values measured and the dashed lines represent the $95 \%$ uncertainty in the mean velocity. Measurement is 2-mm above surface of the flat plate.

and 9c show the tagged profiles for the undelayed and delayed images, respectively. Figure 9d shows the measured average velocity profiles obtained with the cross-correlation algorithm.

Figure 9a shows the flow visualization image at approximately $1.7 \pm 0.3-\mathrm{mm}$ off the model surface. Prior to passing around the cylindrical boundary layer trip, the boundary layer flow is completely laminar. After the flow passes the trip, the region directly behind the trip shows that some level of disturbance is occurring based upon the variation of signal intensities in this image. However, as the flow passes around the trip, a decrease in velocity extends downstream along the centerline, which is observed in Figure 9d. The flow downstream of the upper and 


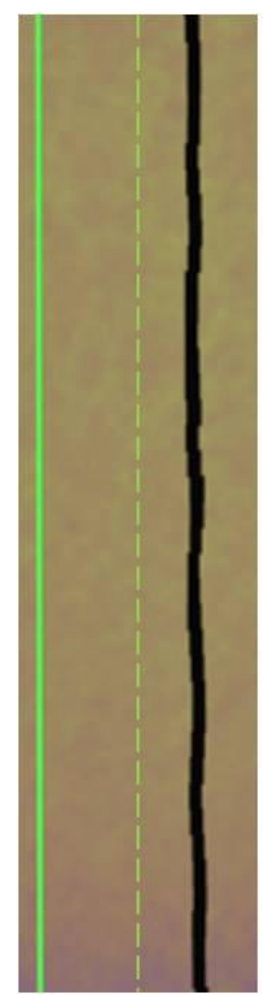

(a)

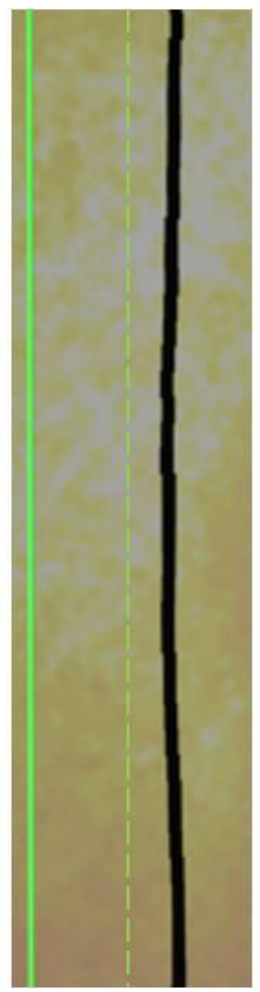

(b)

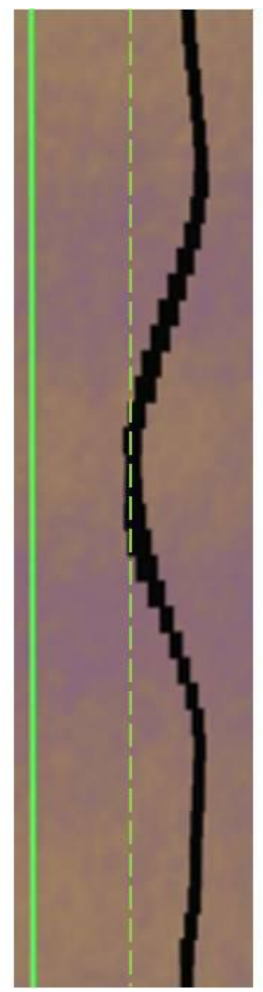

(c)

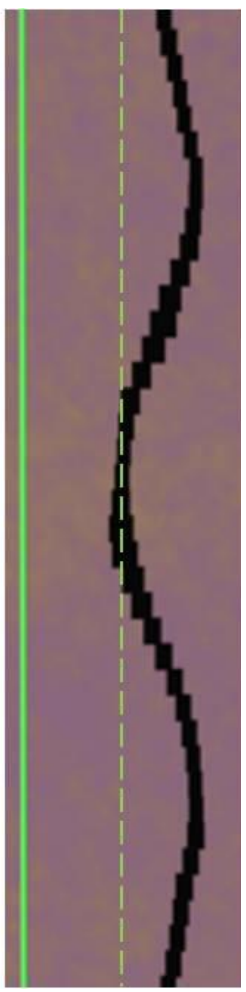

(d)

Figure 10. Magnified view of the mid-portion of the (a) first, (b) fourth, (c) tenth, and (d) twenty-fourth mean velocity profiles and their corresponding uncertainties. The dashed blue line represents the midscale velocity of $630 \mathrm{~m} / \mathrm{s}$.

brought to rest in front of the trip. As the flow progresses around the trip, the measured velocities appear to maintain the pre-trip velocity with the exception of the velocity values in the immediate wake of the trip. Downstream of the trip the velocity observed along the centerline drops to $500 \mathrm{~m} / \mathrm{s}$. In the accelerated flow region directly downstream of the top and bottom edges of the trip, the velocity exceeds $1025 \mathrm{~m} / \mathrm{s}$.

Figure 10 shows from left to right the magnified views of the first, fourth, tenth, and twenty-fourth velocity profiles, respectively. The first profile (Figure 10a) shows a mean velocity of approximately $960 \mathrm{~m} / \mathrm{s}$ with uncertainties between $35-62 \mathrm{~m} / \mathrm{s}$. The decreased velocity feature, a left-bowing profile, in the fourth velocity profile (Figure 10b) can be seen with similar uncertainty magnitudes as those in the first velocity profile. The tenth profile (Figure 10c) shows a drop in velocity along the centerline to approximately $620 \mathrm{~m} / \mathrm{s}$. The increased velocities occurring above and below the low-velocity region are approximately $1025-1050 \mathrm{~m} / \mathrm{s}$. The uncertainty encountered along this profile is slightly larger than that of the unperturbed first profile and varies from $36-75 \mathrm{~m} / \mathrm{s}$. The uncertainty immediately above (with respect to the figure) the lowest velocity value $(\sim 75 \mathrm{~m} / \mathrm{s})$ is greater than that of

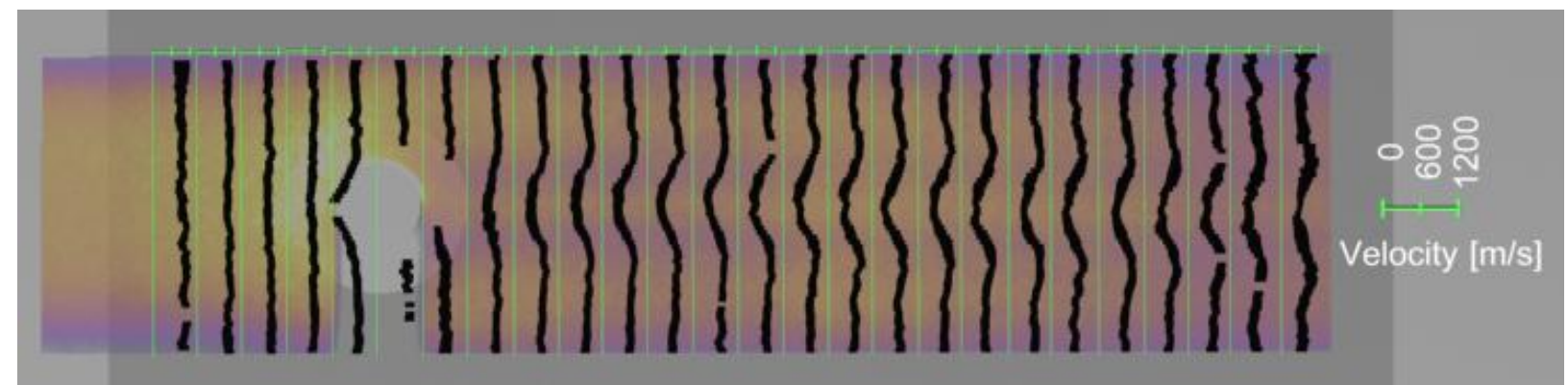

Figure 11. Single-shot velocity profiles and uncertainty bounds. The velocity scale is between $0-1200 \mathrm{~m} / \mathrm{s}$. 
the uncertainty along the remaining portion of the magnified profile, due to the inclusion of the spanwise velocity component uncertainty. The twenty-fourth profile in Figure 10d shows a similar trend to that of the tenth mean velocity profile. The flow above and below the low velocity region, which has a minimum velocity of $604 \mathrm{~m} / \mathrm{s}$, has approximate velocities between 1060-1075 m/s.

Figure 11 shows a single-shot velocity measurement. The decreased signal-to-noise level from the single-shot image results in more variability in the velocity measurements as well as increased uncertainty along each profile. The uncertainties encountered in this single-shot velocity measurement varied from 95-260 m/s.

\section{Discussion}

The double-shutter camera used in both the Orion CEV and wedge experiments served to reduce measurement sensitivity to vibration that would otherwise be encountered in a single-framing camera velocity measurement ${ }^{4}$ where delayed images are compared with an undelayed image separately acquired and uncorrelated in time. The double-shutter camera velocity measurement was also free of errors encountered when using a two-camera velocimetry system, which is also sensitive to vibration. However, the hardware-dependent $500 \mathrm{~ns}$ gate delay between the undelayed and delayed images and the limitation of a single gain setting for both images resulted in the reduced signal levels in the delayed image due to fluorescence decay. To compensate for this decreased signal level, a timing methodology was developed in which the first gate was partially overlapped with the laser pulse so that only a fraction of the fluorescence from the tagged velocity profiles would be acquired in the first exposure, resulting in comparable signal levels in the undelayed and delayed image. Additionally, by varying the length of the second gate, the amount of fluorescence acquired in the second exposure could be controlled to some level. However, by increasing the second gate, the level of blurring and asymmetry of the tagged profile in the second exposure also increased. To account for this, a correction was applied that was dependent upon the signal intensity ratios between the undelayed and delayed profiles as well as the duration of the second gate.

Based upon the measurements observed in this experiment and the simulations performed to determine the correction factor, we determined that using a shorter second gate (100 ns in the wedge experiment) would limit the greater variability encountered in the correction factor if a longer gate (300 ns in the CEV experiment) is employed. One way to avoid the variability due to the correction factor and its associated uncertainty is by generating the profile molecules prior to the start of the first gate via photodissociation. ${ }^{5,18}$ This gas could then be excited prior to and captured by the first and second gates. This method would result in similarly shaped spatial fluorescence profiles, would not require any correction for profile asymmetry, and would remove any uncertainties associated with the overlap of the first gate and the firing of the excitation laser. Another benefit of such a scheme would be the removal of timing dependence on fluorescence lifetime. This would allow for increased temporal separation between the undelayed and delayed images, resulting in decreased uncertainties for those proportional to the separation time, and the ability to work in higher-pressure conditions where fluorescence quenching would otherwise limit the signal in the second gate. Difficulties with this technique include seeding $\mathrm{NO}_{2}$ safely into the flow, the use of higher laser energies in order to photodissociate the $\mathrm{NO}_{2}$, and the heavier molecular weight of the $\mathrm{NO}_{2}$ molecule with respect to air.

A significant contribution to the total velocity uncertainty in both the mean and single-shot velocity measurements was the accuracy measurement. For the average velocity measurement, this in fact was the largest error encountered. For this experiment, the zero-velocity images acquired in the 31-inch Mach 10 wind tunnel were processed to obtain an accuracy estimate. After the CEV and wedge experiments were performed, it was learned that there was the potential for small velocities within the test section when it was reduced to near vacuum due to leaks in the system. If the gas was truly not stagnant within the test section during acquisition of the zero-velocity images, the resulting accuracy value reported herein is larger than necessary. Correction of this may result in lower velocity uncertainties in both mean and single-shot velocity measurements. A possible solution is to have a static cell at the tunnel pressure which can be imaged separately to obtain a measure of velocity accuracy. Such a measure could potentially improve experimental accuracy. If a measurement is being made within a quiescent gas, fluorescence within the zero-flow region can be used to obtain an estimate of velocity accuracy and may also improve accuracy. ${ }^{19}$

Comparison with unpublished work of similar experiments we performed using the undelayed and delayed image capturing method and a single camera acquisition method in quiescent gas, velocity accuracy errors from those experiments have been on the order of more than $200 \mathrm{~m} / \mathrm{s}$. The work reported here represents a factor of ten improvement in the velocity accuracy. 


\section{Conclusion}

This paper describes a velocimetry technique using a single, double-frame camera to obtain spatially and temporally correlated images. A new method was developed to acquire the undelayed and delayed images so that comparable signal intensities would be obtained in both exposures. A correction technique was also introduced which estimated the perceived profile shift observed in the delayed image due to fluorescence decay. Single-shot and mean velocities and uncertainties were calculated for an Orion CEV yaw RCS jet and a 10-degree half-angle wedge in a Mach 10 perfect gas air flow. Errors associated with spanwise velocity (parallel to the laser sheet) were quantified for the first time.

\section{Acknowledgments}

We wish to acknowledge the expert contribution to this work from the 31" Mach 10 tunnel and data system operators at NASA Langley Research Center. This work was supported by NASA's Fundamental Aeronautics Program, Hypersonics Project, Experimental Capabilities and Aero, Aerothermodynamics, and Plasma Discipline.

\section{References}

${ }^{1}$ Danehy, P.M., Wilkes, J.A., Brauckmann, G.J., Alderfer, D.W., Jones, S.B., and Patry, D.P., "Visualization of a Capsule Entry Vehicle Reaction-Control System (RCS) Thruster," AIAA 2006-1532, $44^{\text {th }}$ AIAA Aerospace Sciences Meeting and Exhibit, January 9-12, 2006, Reno, NV.

2 Johnson, S., and Murphy, K., "Pressure-Sensing Performance of Upright Cylinders in a Mach 10 BoundaryLayer," NASA Technical Memorandum 4633, July, 1994.

${ }^{3}$ Koochesfahani, M.M., "Molecular Tagging Velocimetry (MTV): Progress and Applications," AIAA 1999-3786, $30^{\text {th }}$ AIAA Fluid Dynamics Conference, June 28 - July 1, 1999.

${ }^{4}$ Danehy, P.M., O'Byrne, S., Houwing, A.F.P, Fox, J.S., and Smith, D.R., "Flow-Tagging Velocimetry for Hypersonic Flows Using Fluorescence of Nitric Oxide," AIAA Journal, 41 (2), February, 2003.

${ }^{5}$ Hsu, A.G., Srinivasan, R., Bowersox, R.D.W, and North, S.W., "Molecular Tagging Using Vibrationally Excited Nitric Oxide in an Underexpanded Jet Flowfield," AIAA Journal, 47 (11), November, 2009.

${ }^{6}$ Buck, G.M., Watkins, A.N., Danehy, P.M., Inman, J.A., Alderfer, D.W., and Dyakonov, A.A., "Experimental Measurement of RCS Jet Interaction Effects on a Capsule Entry Vehicle," $46^{\text {th }}$ AIAA Aerospace Sciences Meeting and Exhibit, AIAA-2008-1229, Orlando, FL, January 7-10, 2008.

${ }^{7}$ Danehy, P.M., Ivey, C.B., Inman, J.A., Bathel, B.F., Jones, S.B., Jiang, N., Webster, M., Lempert, W., Miller, J., and Meyer, T., "High-speed PLIF imaging of hypersonic transition over discrete cylindrical roughness," AIAA2010-0703, 48 ${ }^{\text {th }}$ AIAA Aerospace Sciences Meeting, Orlando, FL, January 4-7, 2010.

${ }^{8}$ Danehy, P.M., Bathel, B.F., Ivey, C., Inman, J.A., and Jones, S.B., "NO PLIF study of hypersonic transition over a discrete hemispherical roughness element," AIAA 2009-394, $47^{\text {th }}$ AIAA Aerospace Sciences Meeting, January 5-8, 2009, Orlando, Florida.

${ }^{9}$ Gendrich, C.P, and Koochesfahani, M.M., "A spatial correlation technique for estimating velocity fields using molecular tagging velocimetry (MTV)," Experiments in Fluids, Vol. 22, pp. 67-77, 1996.

${ }^{10}$ Micol, J.R., "Langley Aerothermodynamic Facilities Complex: Enchancements and Testing Capabilities," AIAA 1998-0147, $36^{\text {th }}$ AIAA Aerospace Sciences Meeting, January 12-15, 1998, Reno, Nevada.

${ }^{11}$ Berry, S.A., Nowak, R.J., and Horvath, T.J., "Boundary Layer Control for Hypersonic Airbreathing Vehicles," AIAA 2004-2246, $34^{\text {th }}$ AIAA Fluid Dynamics Conference and Exhibit, June 28 - July 1, 2004, Portland, Oregon, 2004.

${ }^{12}$ Alderfer, D.W., Danehy, P.M., Wilkes Inman, J.A., Berger, K.T., Buck, G.M., and Schwartz, R.J., "Fluorescence Visualization of Hypersonic Flow Over Rapid Prototype Wind-Tunnel Models," AIAA 2007-1063, 45 ${ }^{\text {th }}$ Aerospace Sciences Meeting and Exhibit, Reno, NV, January 8-11, 2007.

${ }^{13}$ Sanchez Sorzano, C.O., Thevenaz, P., and Unser, M., "Elastic Registration of Biological Images Using VectorSpline Regularization," IEEE Transactions on Biomedical Engineering, 52 (4), pp. 652-663, April, 2005.

${ }^{14}$ Rasband, W.S., ImageJ, U. S. National Institutes of Health, Bethesda, MD, USA, http://rsb.info.nih.gov/ij, 19972009.

${ }^{15}$ Settersten, T.B., Patterson, B.D., and Carter, C.D., "Collisional quenching of $N O A^{2} \Sigma^{+}\left(v^{\prime}=0\right)$ between 125 and 294 K," Journal of Chemical Physics, Vol. 130, 2009.

${ }^{16}$ Settersten, T.B., Patterson, B.D., and Humpries IV, W.H., "Radiative lifetimes of $N O A^{2} \Sigma^{+}\left(v^{\prime}=0,1,2\right)$ and the electronic transition moment of the $A^{2} \Sigma^{+}-X^{2} \Pi$ system," Journal of Chemical Physics, Vol. 131, 2009. 
${ }^{17}$ Schwartz, R.J., "ViDI: Virtual Diagnostics Interface Volume 1-The Future of Wind Tunnel Testing," Contractor Report NASA/CR-2003-212667, December, 2003.

${ }^{18}$ Hsu, A.G., Srinivasan, R., Bowersox, R.D.W., and North, S.W., "Two-component molecular tagging velocimetry utilizing $\mathrm{NO}$ fluorescence lifetime and $\mathrm{NO}_{2}$ photodissociation techniques in an underexpanded jet flowfield," Optics Letter, 48 (22), August, 2009.

${ }^{19}$ Inman, J.A., Danehy, P.M., Bathel, B.F., and Nowak, R.J., "Laser-Induced Fluorescence Velocity Measurements in Supersonic Underexpanded Impinging Jets," $48^{\text {th }}$ AIAA Aerospace Sciences Meeting and Exhibit, AIAA-20101438, Orlando, FL, January 3-7, 2010. 